\title{
Twisted Catheter Causing Baclofen Pump Malfunction: A Case Report
}

\author{
Lyndsay J. Russell, Andre A. leRoux, W. Brian Wheelock
}

Can J Neurol Sci. 2012; 39: 838-839

A 49-year-old man presented with a history of multiple sclerosis, causing him increased tone and spasticity in the lower extremities. This severely affected his ambulation and mobility. Following the titration of oral baclofen and tizanidine to maximum tolerated doses with little impact on his spasticity, the patient agreed to a trial dose of $50 \mu \mathrm{g}$ of intrathecal baclofen (ITB), which was effective in reducing his spasticity and improving mobility. He subsequently underwent implantation of a SynchroMed II (Medtronic, Minneapolis, Minn) ITB pump, with the tip of the catheter placed at the T10/T11 junction, and the pump placed in the right upper quadrant of his abdomen. The patient's spasticity and mobility improved on a small daily dose of ITB, 500 micrograms per $\mathrm{ml}(91 \mu \mathrm{g}$ /day). He lived at a distance from the center, and appeared to be doing well with the small dose, and therefore had not had any further adjustments. Six months postoperatively, he noticed return of the spasms. His baclofen dose was increased to $103.95 \mu \mathrm{g}$ /day. Three months later, he presented with a sudden recurrence of severe spasticity. The baclofen was increased to $120 \mu \mathrm{g}$ /day without improvement. On his scheduled refill visit, $19 \mathrm{ml}$ of baclofen was aspirated instead of the anticipated $3 \mathrm{ml}$.

In the neurointerventional suite we were unable to aspirate fluid from the catheter access port (CAP). Several loops of catheter were seen below the pump, any of which could potentially be kinked (Figure 1). A decision was made to revise the pump and catheter. The patient received oral baclofen during the interval between the interventional and surgical procedures. During surgery it was discovered that the pump sutures had broken, the pump had overturned within the abdominal pocket, and the pump catheter was in a large knot (Figure 2). The catheter was unraveled by turning the pump 23 times. Despite straightening the pump catheter, we were still unable to aspirate

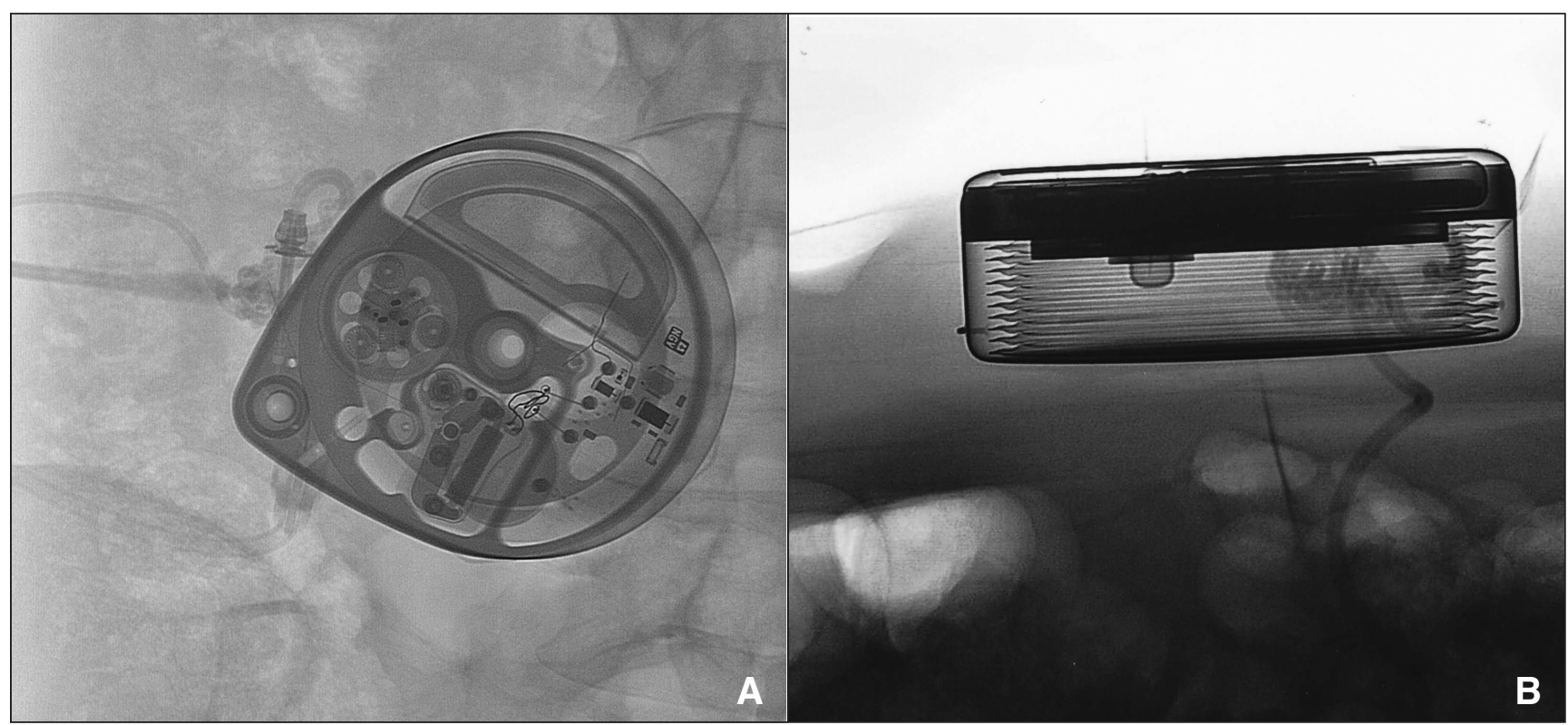

Figure 1: Fluoroscopy prior to surgical revision showing knotted loops of catheter below the pump: anteroposterior (A) and lateral (B).

From Dalhousie Medicine New Brunswick, Saint John, New Brunswick, Canada.

Received June 1, 2012. Final Revisions Submitted June 25, 2012.

Correspondence to: W. Brian Wheelock, 3BN, Department of Neurosurgery, Saint John Regional Hospital, 400 University Avenue, Saint John, New Brunswick,

E2L 4L2, Canada. Email: brian.wheelock@horizonnb.ca. 
fluid from the CAP. The back incision was opened, and the spinal catheter was also found to have twisted a number of times. CSF flow was restored when the spinal catheter was unraveled. It was trimmed and connected to a new pump catheter, and the pump was repositioned in the abdominal pocket.

\section{Discussion}

Despite their efficacy in controlling severe spasticity, ITB pump systems are subject to complications. The catheter has consistently been documented as the most vulnerable part of the system. ${ }^{1-3}$ A systematic review found 558 reported complications out of 1362 pump implants, with $66 \%$ related to catheter malfunctions. Common catheter complications include breaks, cuts, disconnections, dislodgements, fibrosis, holes, kinks and occlusions. ${ }^{1}$ However, pump flipping leading to catheter twisting and occlusion is a very uncommon complication. ${ }^{4}$ The need to enlarge the pocket to place the tie-down sutures can predispose the pump to flipping if the sutures erode or pull through the fascia. Fukuharaet al described a case of a tangled catheter causing baclofen pump malfunction, which to their knowledge had not previously been documented in the literature. ${ }^{5}$ However, unlike the present case, their patient's catheter became twisted without the pump having rotated within the pocket.

In our case, we believe that the breaking of the sutures predisposed the pump to flip within an oversized abdominal pocket. The patient was completing self-transfers, which may have provided the additional torque needed to flip the pump 23 times. The flipping of the pump caused the catheter to twist, leading to the occlusion of its lumen. Our belief is that multiple catheter twists were transmitted downstream, resulting in a secondary knot in the spinal catheter. This prevented the delivery of the ITB to the CSF, causing the patient to experience symptoms of ITB withdrawal.

\section{Prevention}

A number of techniques have been suggested to reduce the risk of pump flipping. The pump will have less room to overturn if the size of the incision and the volume of the abdominal pocket are limited. The pump must be properly anchored within the abdominal pocket, either with permanent sutures placed around the pump, or by placing the pump in a mesh, which encourages scar formation. ${ }^{4}$ Alternative consideration can be given to a design change to incorporate a suture loop at the inferior center of the pump.

\section{CONCLUSION}

Twisting of the catheter is a rare complication of ITB pump systems. It is important that physicians be aware that it is a potential complication and recognize the signs of pump malfunction. It is possible to decrease the risk of pump rotation by reducing the size of the incision and abdominal pocket volume, and ensuring proper anchoring of the pump within the pocket.

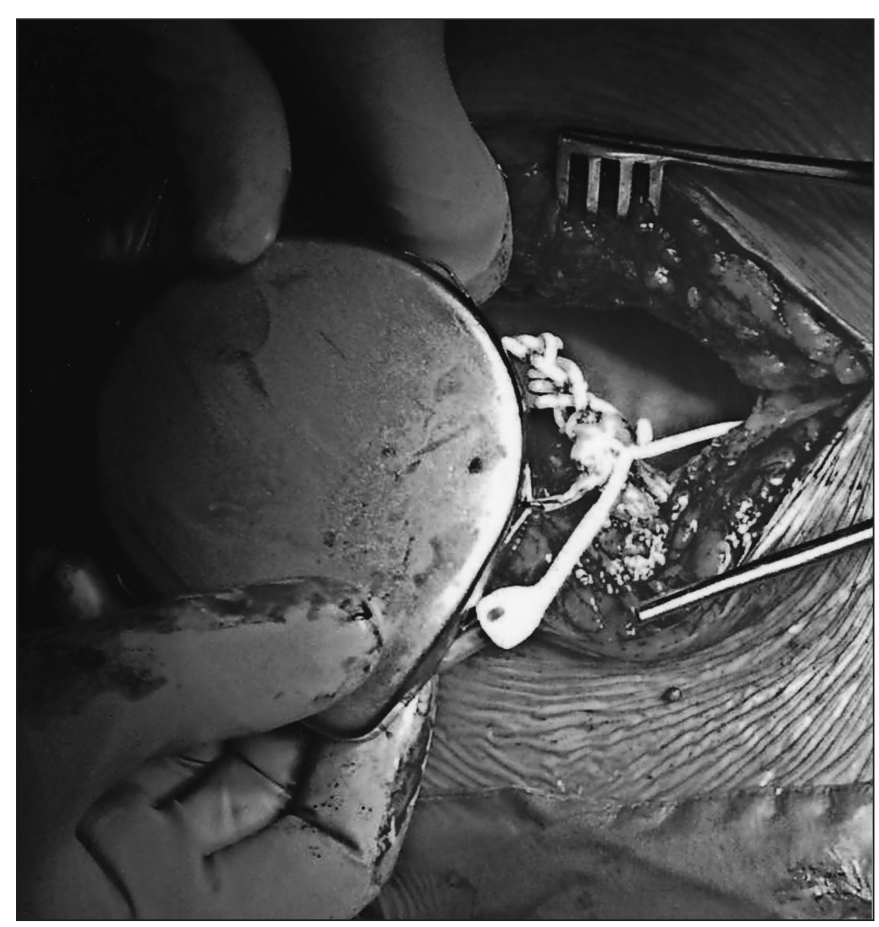

Figure 2: The pump catheter was found in a large knot during surgical revision.

\section{ACKNOWLEDGEMENTS}

The authors thank the Neurosurgeons of the Saint John Regional Hospital for all of their wonderful teaching and support during their preceptorship, and Kathleen Gadd for her editing help.

\section{REFERENCES}

1. Stetkarova I, Yablon SA, Kofler M, Stokic DS. Procedure-and device-related complications of intrathecal baclofen administration for management of adult muscle hypertonia: a review. Neurorehab Neural Repair. 2010;24(7):609-19.

2. Flückiger B, Knecht H, Grossmann S, Felleiter P. Device-related complications of long-term intrathecal drug therapy via implanted pumps. Spinal Cord. 2008;46:639-43.

3. Li TC, Chen MH, Huang JS, Chan JY, Liu YK, Chen MH. Catheter migration after implantation of an intrathecal baclofen infusion pump for severe spasticity: a case report. Kaohsiung J Med Sci. 2008;24(9):492-6.

4. Vender JR, Hester S, Rekito A, Lee MR. Baclofen intrathecal pump delivery systems: avoidance and management of complications in adult and pediatric patients: Part I. Contemp Neurosurg. 2005; 27(1):1-7.

5. Fukuhara T, Tanaka T, Namba Y, Kuyama H. Tangled catheter as a rare cause of baclofen pump malfunction. Surg Neurol. 2009;72: 80-2. 